POS PROCEEDINGS

\title{
Searches for supersymmetric gaugino production in final states with leptons with the ATLAS detector
}

\section{Tobias Kruker*}

On behalf of the ATLAS Collaboration

Albert Einstein Center for Fundamental Physics

Laboratory for High Energy Physics

University of Bern, Sidlerstrasse 5, 3012 Bern, Switzerland

E-mail: kruker@gmx.ch

\begin{abstract}
Searches for electroweakly produced sleptons, charginos and neutralinos in final states with two and three electrons or muons are presented. The searches are performed on $4.7 \mathrm{fb}^{-1}$ of $\sqrt{s}=7$ $\mathrm{TeV}$ proton-proton collision data recorded with the ATLAS detector at the Large Hadron Collider. No significant excess is found in any of the searches. The results are interpreted in the framework of the phenomenological minimal supersymmetric standard model and simplified models.
\end{abstract}

36th International Conference on High Energy Physics

4-11 July 2012

Melbourne, Australia

\footnotetext{
* Speaker.
} 


\section{Introduction}

Supersymmetry (SUSY) is an extension of the standard model (SM) that postulates partners to all SM particles. The spin of the SUSY particles differs by one half unit from the corresponding SM partners. The scalar partners of the charged SM leptons are called sleptons and are denoted $\tilde{\ell}^{ \pm}$, the partners of the neutrinos are the sneutrinos $\tilde{v}$. Gluinos and squarks are the SUSY partners of the gluons and quarks. The SUSY partners of the gauge bosons and Higgs particles carry spin $1 / 2$ and are called gauginos and higgsinos, respectively. In the MSSM, they form the mass eigenstates charginos $\left(\tilde{\chi}_{i}^{ \pm}, i=1,2\right)$ and neutralinos $\left(\tilde{\chi}_{i}^{0}, i=1,2,3,4\right)$.

In models that assume very massive (greater than a few $\mathrm{TeV}$ ) gluinos and squarks, the direct production of slepton and chargino/neutralino pairs is the dominant SUSY production mode at the Large Hadron Collider (LHC). The decay of these particles can lead to high lepton multiplicities. The searches presented here target final states with exactly two leptons (two lepton analysis) and exactly three leptons (three lepton analysis). Here, lepton refers to electrons and muons. The decay modes of interest of the sleptons, charginos, and neutralinos are $\tilde{\ell}^{ \pm} \rightarrow \ell^{ \pm} \tilde{\chi}_{1}^{0}, \tilde{\chi}_{1}^{ \pm} \rightarrow \ell^{ \pm} v \tilde{\chi}_{1}^{0}$, $\tilde{\chi}_{2}^{0} \rightarrow \ell^{ \pm} \ell^{\mp} \tilde{\chi}_{1}^{0}$, respectively.

Both analyses make use of the full $2011 \sqrt{s}=7 \mathrm{TeV}$ proton-proton collision dataset recorded with the ATLAS detector at the LHC. The dataset corresponds to an integrated luminosity of 4.7 $\mathrm{fb}^{-1}$ (after applying data quality requirements). Both analyses are submitted for publication: two lepton analysis [1], three lepton analysis [2]. The ATLAS experiment is described in [3].

\section{Searches for electroweakly produced sleptons, charginos, and neutralinos in final states with 2 and 3 leptons}

\subsection{Signal models}

The optimization of the searches and the statistical interpretation of the results are performed in phenomenological minimal supersymmetric standard models (pMSSM) and simplified models.

The model used for the direct slepton (DS) search is based on the pMSSM in [4]. The masses of the charginos and neutralinos (except for the $\tilde{\chi}_{1}^{0}$ ) are set to $2.5 \mathrm{TeV}$. Degenerate selectron and smuon masses are assumed and are generated under the constraint $m_{\tilde{\ell}}>m_{\tilde{\chi}_{1}^{0}}+30 \mathrm{GeV}$. The model is scanned as a function of the slepton and the $\tilde{\chi}_{1}^{0}$ masses.

In the pMSSM models [5] used for the search for chargino/neutralino pair production, the production cross sections are governed by the gaugino mass parameters $M_{1}$ and $M_{2}$, the Higgshiggsino mass parameter $\mu$, and the ratio of the vacuum expectation values of the two Higgs doublet fields $\tan \beta$. The masses of the squarks, gluinos, and left-handed sleptons (SUSY partners of the left-handed leptons) are set to values greater than $2 \mathrm{TeV}$. The models are investigated for three different $M_{1}$ choices and scanned as a function of $\mu$ and $M_{2}$.

Two different pair production modes are considered in the framework of the simplified models [6]: the chargino-neutralino pair production (Mode A, $\tilde{\chi}_{1}^{ \pm} \tilde{\chi}_{2}^{0}$ ) and the chargino pair production (Mode $\mathrm{C}, \tilde{\chi}_{1}^{ \pm} \tilde{\chi}_{1}^{\mp}$ ). The following choices for the masses of the involved particles are made: $m_{\tilde{\chi}_{2}^{0}}=$ $m_{\tilde{\chi}_{1}^{ \pm}}, m_{\tilde{v}}=m_{\tilde{\ell}_{\mathrm{L}}}=\left(m_{\tilde{\chi}_{1}^{0}}+m_{\tilde{\chi}_{1}^{\mp}}\right) / 2$. The models are investigated for the only free parameters left, the $\tilde{\chi}_{1}^{ \pm}$and $\tilde{\chi}_{1}^{0}$ masses. The $\tilde{\chi}_{1}^{ \pm}$and $\tilde{\chi}_{2}^{0}$ decay via sneutrinos and left handed sleptons with a branching ratio of $50 \%$ each. 
In all models, $R$-parity is conserved and the $\tilde{\chi}_{1}^{0}$ is the lightest SUSY particle (LSP). The results from the two lepton analysis are used for the DS search and the interpretation in the Mode C of the simplified models. The three lepton analysis is more sensitive to chargino/neutralino pair production in the pMSSM and Mode A of the simplified models.

\subsection{Two lepton analysis}

Four signal regions (SR) were optimized for the DS search and different chargino / neutralino decay modes. SR- $m_{\mathrm{T} 2}$ is the main SR and brings good sensitivity for DS and chargino pair production processes. Differences in event topologies between signal and background (BG) events are exploited using the stransverse mass [7, 8] $m_{\mathrm{T} 2}$, where $\max \left[m_{\mathrm{T}}\left(\mathbf{p}_{\mathrm{T}}^{\ell_{1}}, \mathbf{q}_{\mathrm{T}}\right), m_{\mathrm{T}}\left(\mathbf{p}_{\mathrm{T}}^{\ell_{2}}, \mathbf{r}_{\mathrm{T}}\right)\right]$ is minimized under the constraint $\mathbf{q}_{\mathrm{T}}+\mathbf{r}_{\mathrm{T}}=\mathbf{p}_{\mathrm{T}}^{\text {miss }} \cdot \mathbf{p}_{\mathrm{T}}^{\ell_{1}}$ and $\mathbf{p}_{\mathrm{T}}^{\ell_{2}}$ are the transverse momenta of the two leptons and $\mathbf{q}_{\mathrm{T}}, \mathbf{r}_{\mathrm{T}}$ are introduced as placeholders for the two undetectable LSP. The transverse mass $m_{\mathrm{T}}$ is the mass of a two particle system computed in the transverse plain. A requirement of $m_{\mathrm{T} 2}>90 \mathrm{GeV}$ is applied.

The main SM BG processes in this region are top pair $(t \bar{t})$ and single top production, $Z / \gamma^{*}+$ jets, and dibosons $(W W, W Z, Z Z)$. The $\mathrm{BG}$ from these processes is estimated through Monte Carlo (MC) simulation combined with information from data. The reducible "fake lepton" BG where jets or photon conversions are mis-identified as prompt leptons is obtained from a data-driven matrix method (MM). The MM exploits differences in object characteristics between real and fake leptons. Events are categorized according to the lepton identification quality. Identification efficiencies and fake rates are used to obtain the fake contribution in SR.

The selection criteria of the four signal regions SR- $m_{\mathrm{T} 2}$, SR-OSjveto, SR-SSjveto, and SR2 jets as well as the BG estimation techniques are described in detail in [1].

\subsection{Three lepton analysis}

Three signal regions are defined in the three lepton analysis, two of which are used for the interpretation of the results in the pMSSM and simplified models Mode A. The main SR called SR1a targets decays via intermediate sleptons or off-shell bosons, e.g. $\tilde{\chi}_{2}^{0} \rightarrow \tilde{\ell} \bar{\ell} \rightarrow \ell \bar{\ell} \tilde{\chi}_{1}^{0}$. Events with $Z$-boson candidates are therefore rejected. The two main additional requirements of SR1a are: $b$-jet veto and $E_{\mathrm{T}}^{\text {miss }}>75 \mathrm{GeV}$. SR $1 \mathrm{~b}$ is a tighter version of SR1a and gives additional sensitivity to larger mass splittings in the SUSY particle mass spectrum. The third SR (SR2) complements the search region by requiring a $Z$-boson candidate.

The main BG in SR1a are $W Z$ dibosons and $t \bar{t} . t \bar{t}$ only contributes if at least one lepton is a fake. $t \bar{t}$ (along with all other reducible BG) is estimated using a similar MM technique as in the two lepton analysis. $W Z$ is normalized to data in a dedicated region in phase space. For limit setting purposes, the signal contamination in this region is taken into account. The other irreducible (three real leptons) BG are estimated from MC simulation.

The selection criteria of the three signal regions and the BG estimation techniques are described in detail in [2]. 


\section{Results}

Table 1 lists data observations and the total BG expectations including the systematic uncertainties in the four SR of the two lepton analysis and the three SR of the three lepton analysis. For SR- $m_{\mathrm{T} 2}$ also the same flavor (SF) channel is shown because this channel is used in the DS search. No significant excess of data over the expected SM BG is observed in any of the regions. The searches have not found any sign for DS, $\tilde{\chi}_{1}^{ \pm} \tilde{\chi}_{1}^{\mp}$, or $\tilde{\chi}_{1}^{ \pm} \tilde{\chi}_{2}^{0}$ production.

The expected and observed exclusion limits at $95 \%$ confidence level based on the $\mathrm{CL}_{s}$ method [9] are presented in Figures 1 through 3. They are calculated without signal theory uncertainty. The yellow band is the $\pm 1 \sigma$ experimental uncertainty on the expected limit (black dashed line). The red dashed band is the $\pm 1 \sigma$ signal theory uncertainty on the observed limit (red solid line). The orange shaded areas indicate the LEP limits. In Figure 1 (a) the LEP limit [10] is on the mass of the right-handed smuon. The LEP2 limits in the other plots correspond to the limit on the $\tilde{\chi}_{1}^{ \pm}$mass in [11] as transposed to the corresponding plane.

The limits in Figure 1 (a) are the first ATLAS limits on direct slepton production. A common value for left-handed selectron and left-handed smuon mass between 85 and $195 \mathrm{GeV}$ is excluded when the lightest neutralino has a mass of $20 \mathrm{GeV}$. The limits in the pMSSM with $M_{1}=100 \mathrm{GeV}$ (Figure 1 (b)) are significantly extended with respect to the previous analysis based on $2 \mathrm{fb}^{-1}$ [12], while the limits in Figure 2 on $M_{1}=140 \mathrm{GeV}$ (a) and $250 \mathrm{GeV}$ (b) are first results on these models.

The limits in the simplified models in Mode A (Figure 3 (a)) significantly increase the limits from the preceding analysis based on $2 \mathrm{fb}^{-1}$ [12]. The limits in Mode $\mathrm{C}$ (chargino pair production, Figure 3 (b)) are first ATLAS results in this Mode and exclude chargino masses between 110 and $340 \mathrm{GeV}$ for a $10 \mathrm{GeV}$ neutralino.

\section{Conclusions}

Searches for electroweakly produced sleptons, charginos and neutralinos in final states with two and three electrons or muons have been presented. The searches probe the electroweak SUSY production at the LHC. No significant excess above the SM BG is observed. First ATLAS limits on direct slepton and direct chargino pair production are presented and limits on direct chargino/neutralino pair production are extended significantly with respect to previous analyses.

\section{References}

[1] ATLAS Collaboration, arXiv:1208.2884 [hep-ex]

[2] ATLAS Collaboration, arXiv:1208.3144 [hep-ex]

[3] ATLAS Collaboration, 2008 JINST 3 S08003

[4] S. S. AbdusSalam et al., Eur. Phys. J. C58 (2011) 1835, [arXiv:1109.3859 [hep-ph]]

[5] A. Djouadi, J.L. Kneur, G. Moultaka, Comput. Phys. Commun. 176 (2007) 426, [hep-ph/0211331]

[6] J. Alwall, P. Schuster, N. Toro, Phys. Rev. D 79 (2009) 075020, [arXiv:0810.3921]

[7] A. Barr, C. Lester, and P. Stephens, J. Phys. G G29 (2003) 2343-2363, [arXiv:hep-ph/0304226]

[8] C. Lester and D. Summers, Phys. Lett. B463 (1999) 99-103, [arXiv:hep-ph/9906349] 
Searches for supersymmetric gaugino production in final states with leptons with the ATLAS detector Tobias Kruker

[9] A.L. Read, Presentation of search results: The CL(s) technique J. Phys., G28, 2002, 2693-2704

[10] LEP SUSY Working Group (ALEPH, DELPHI, L3, OPAL), Notes LEPSUSYWG/01-03.1 and 04-01.1, http: / / lepsusy.web.cern.ch/lepsusy/Welcome.html

[11] K. Nakamura et al. (Particle Data Group), J. Phys. G 37 (2010) 075021

[12] ATLAS Collaboration, Phys. Rev. Lett. 108 (2012) 261804, 536 [arXiv.org:1204.5638]

\begin{tabular}{|c|c|c|c|c|c|}
\hline 2 Lep. & SR-m $\mathrm{T} 2$ all & $\mathbf{S R}-\mathbf{m}_{\mathrm{T} 2} \mathbf{S F}$ & SR-OSjveto & SR-SSjveto & SR-2jets \\
\hline BG & $32.8 \pm 3.2 \pm 6.3$ & $20.4 \pm 2.6 \pm 3.9$ & $161.7 \pm 6.7 \pm 30.8$ & $11.0 \pm 1.5 \pm 3.9$ & $65.5 \pm 4.0 \pm 31.8$ \\
\hline Data & 24 & 15 & 139 & 9 & 78 \\
\hline 3 Lep. & SR1a & SR1b & SR2 & & \\
\hline $\begin{array}{l}\text { BG } \\
\text { Data }\end{array}$ & $\begin{array}{c}25 \pm 6 \\
24\end{array}$ & $\begin{array}{c}1.6 \pm 0.5 \\
0\end{array}$ & $\begin{array}{c}10.9 \pm 2.4 \\
11\end{array}$ & & \\
\hline
\end{tabular}

Table 1: BG expectations and data observations in the two lepton analysis signal regions [1] (top) and the three lepton analysis signal regions [2] (bottom). For the two lepton SR the uncertainties from limited MC statistics and systematic uncertainties are shown separately (in this order).

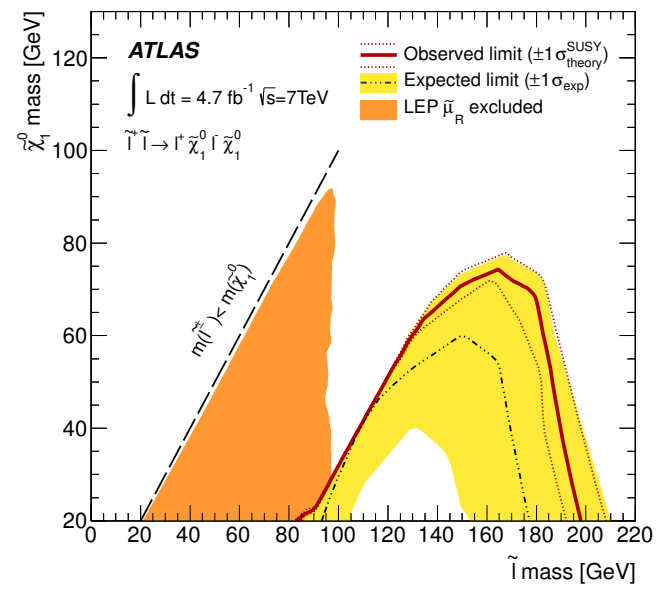

(a)

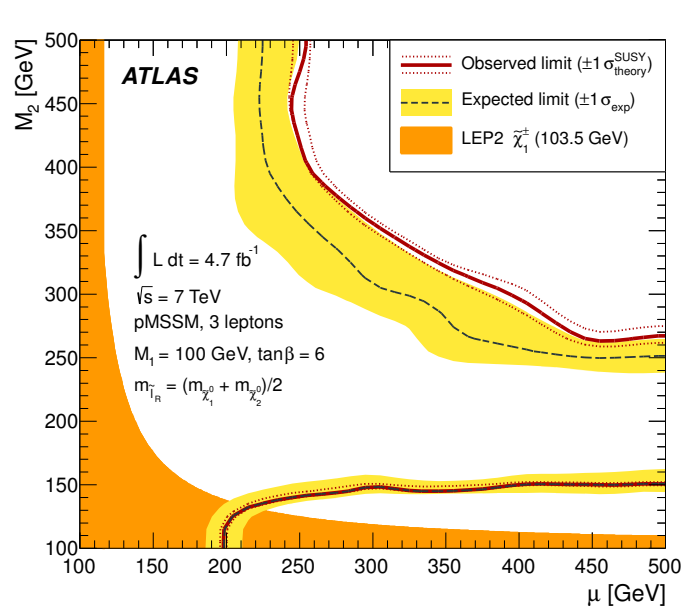

(b)

Figure 1: (a) Observed and expected exclusion contours for slepton pair production in the $\tilde{\ell}-\tilde{\chi}_{1}^{0}$ mass plane of the direct slepton pMSSM as obtained in the two lepton analysis from SR- $m_{\mathrm{T} 2}$ in the same flavor channel [1]. (b) Observed and expected exclusion contours for chargino and neutralino production in the pMSSM for $M_{1}=100 \mathrm{GeV}$ from the three lepton analysis [2]. The exclusion contours are optimized by applying in each signal grid point the $\mathrm{CL}_{s}$ values from the most sensitive signal region. 


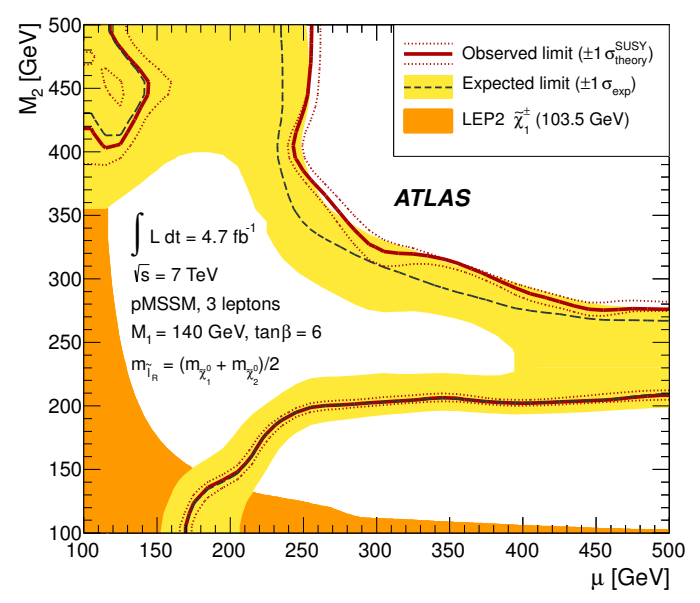

(a)

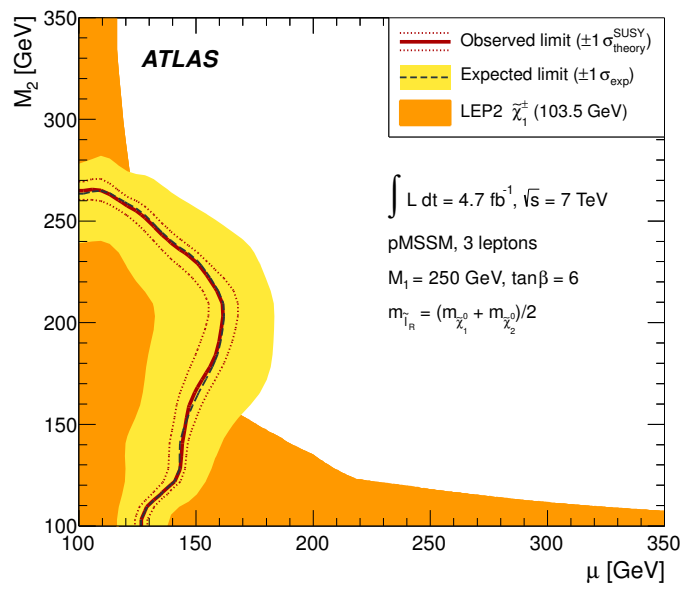

(b)

Figure 2: Observed and expected exclusion contours for chargino and neutralino production in the pMSSM for $M_{1}=140 \mathrm{GeV}$ (a) and $M_{1}=250 \mathrm{GeV}$ (b) from the three lepton analysis [2]. The regions with low values of $M_{2}$ and $\mu$ are excluded. The exclusion contours in (a) are optimized by applying in each signal grid point the $\mathrm{CL}_{s}$ values from the most sensitive signal region while the contour is from SR1a in (b).

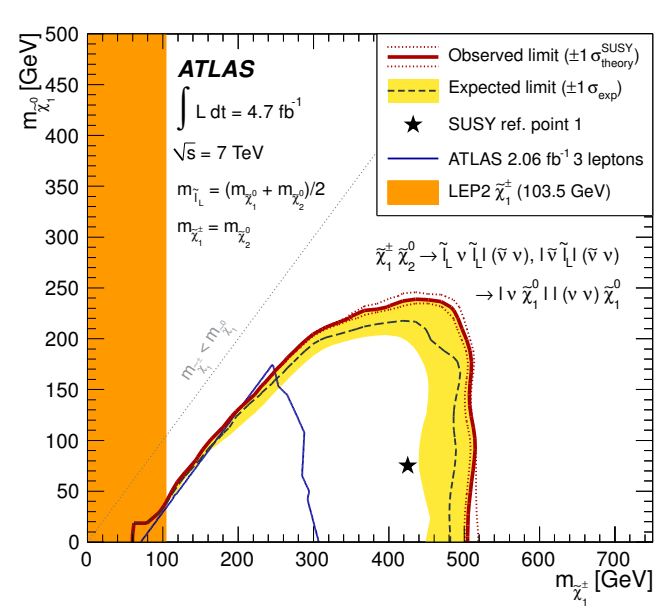

(a)

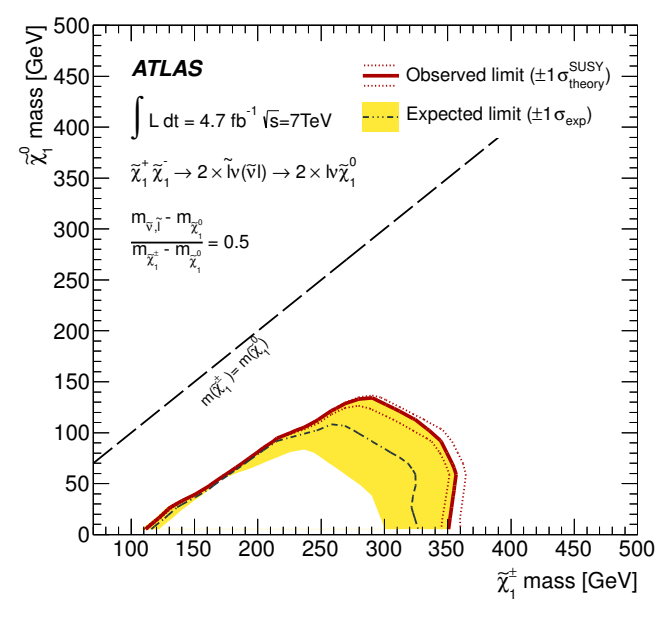

(b)

Figure 3: (a) Observed and expected exclusion contours in the simplified models. (a) shows the scenario Mode A ( $\tilde{\chi}_{1}^{ \pm} \tilde{\chi}_{2}^{0}$ pair production) from the three lepton analysis (SR1b) [2], (b) shows the scenario Mode $\mathrm{C}\left(\tilde{\chi}_{1}^{ \pm} \tilde{\chi}_{1}^{\mp}\right.$ pair production) from the two lepton analysis [1]. The "ATLAS $2.06 \mathrm{fb}^{-1} 3$ leptons" contour in (a) corresponds to the result of the ATLAS search documented in [12]. In (b) the exclusion contours are optimized by applying in each signal grid point the $\mathrm{CL}_{s}$ values from the most sensitive signal region. 